\title{
Spatially Resolved Electron Energy Loss Spectroscopy Studies in Graphene Liquid Cell for the Investigation of the Biomineralization Processes in Human Body
}

\author{
Emre Firlar ${ }^{1,2}$, Kun $\mathrm{He}^{1}$, Reza Shahbazian-Yassar ${ }^{1}$, Tolou Shokuhfar ${ }^{2}$, \\ ${ }^{1 .}$ University of Illinois at Chicago, Department of Industrial and Mechanical Engineering, Chicago IL, \\ USA \\ 2. University of Illinois at Chicago, Department of Bioengineering, Chicago IL, USA
}

Biomineralization of matter in human body has been of a great interest for the biological, medical and materials scientists for many years. The motivation for this type of work remained to be mostly the similar, specifically, after the fully understanding of the biomineralization routes for these minerals, it has been aimed that 1) These minerals could be synthesized in vitro and 2) The cures to the diseases related with these minerals could be developed. Several different minerals with regard to the investigation of biomineralization routes have been studied during the past few decades, for instance, ferritins [1], hydroxyapatite [2], and magnetite [3]. The major challenges for the investigation of these biomineralization processes remained two fold, either not simulating the native environment of these minerals forming or not using the instruments with proper atomic resolution imaging and characterization capabilities. To that end, our approach for the investigation of biomineralization involves the utilization of the Graphene Liquid Cell (GLC) in Cs corrected Scanning Transmission Electron Microscope (STEM) at $80 \mathrm{kV}$. Encapsulating the liquid media in GLC will help to obtain high resolution in both TEM and STEM imaging [4]. With the help of spatially resolved Electron Energy Loss Spectroscopy (EELS) and High Resolution Transmission Electron Microscopy (HRTEM) images, the chemistry of the mineral and structure changes will be visualized, respectively. The advantage of using GLC instead of cryogenic and in situ liquid cell imaging is that, the imaging will be dynamic and due to less sample thickness, analytical TEM studies will be easier, respectively. In this work, we carried out hydroxyapatite biomineralization experiments in GLC so as to have a deep understanding of this biomineralization process. The procedure to encapsulate liquid media and biological structures is as described in Shokuhfar et al. [5]. The chemical information from these minerals with regard to $\mathrm{Ca}_{2,3}, \mathrm{P}$ $\mathrm{L}_{2,3}$ and $\mathrm{O} \mathrm{K}$ edge changes with respect to the total electron dose will be reported via the spatially resolved EELS in HAADF-STEM mode. The formation of this mineral is seen in the inset of Figure 1. The EEL spectrum from the same particle verifies the formation of the calcium phosphate mineral with the presence of the fingerprints of $\mathrm{Ca} \mathrm{L}$ edge, in conjunction to the $\mathrm{C} \mathrm{K}$ edge from the graphene layer [6].

References:

[1] M. Allen et al.: Protein cage constrained synthesis of ferrimagnetic iron oxide nanoparticles Advanced Materials. 14, 21, (2002), pp. 1562-1565.

[2] M.J. Olszta et al.: Bone structure and formation: A new perspective Materials Science and Engineering: R: Reports. 58, 3-5, (2007), pp. 77-116.

[3] J.L. Kirschvink et al.: Magnetite biomineralization in the human brain Proceedings of the National Academy of Sciences. 89, 16, (1992), pp. 7683-7687.

[4] J.M. Yuk et al.: High-resolution EM of colloidal nanocrystal growth using graphene liquid cells Science. 336(6077), 61 (2012), pp. 61-64. 
[5] C. Wang et al.: High resolution electron microscopy and spectroscopy of ferritin in biocompatible liquid cells and graphene sandwiches, Advanced Materials, 26, 3410, pp. 3410-3414.

[6] This project is funded by National Science Foundation- CAREER award (Grant No - DMR0959470). This work made use of instruments in the Electron Microscopy Service (Research Resources Center, UIC)

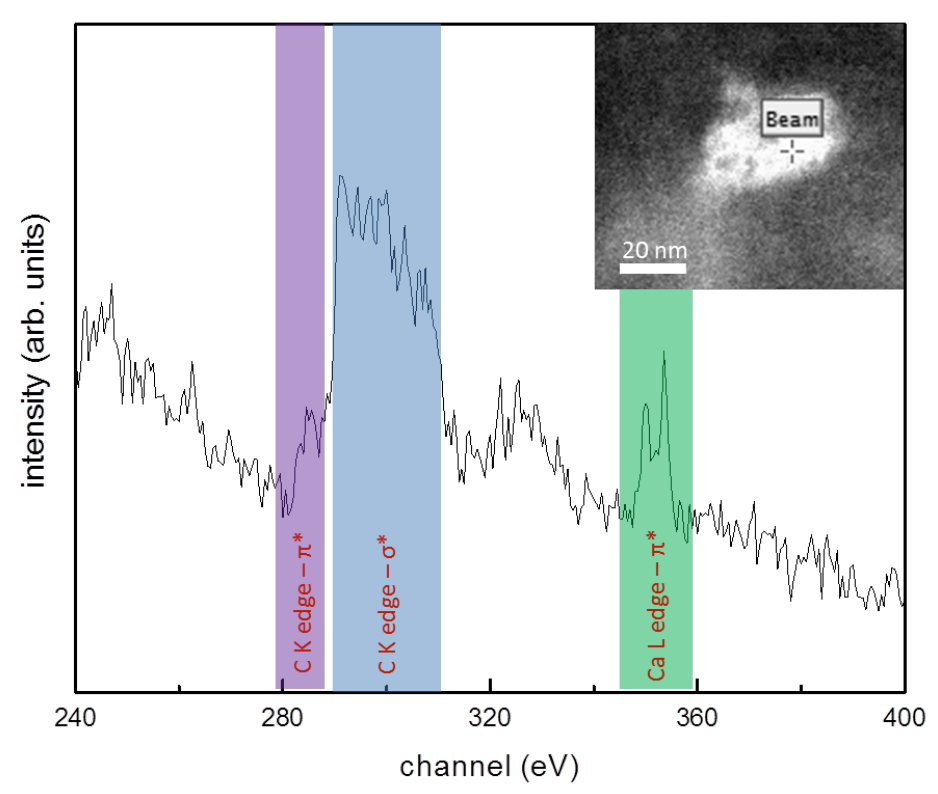

Figure 1. Spatially resolved EELS from a calcium phosphate particle showing Ca L edge from the mineral and $\mathrm{C} \mathrm{K}$ edge from the graphene. Inset: Reference HAADF STEM image showing the calcium oxide mineral formed. 